\title{
Screening swabs surpass traditional risk factors as predictors of MRSA bacteremia
}

\author{
Guillaume Butler-Laporte ${ }^{1^{*}}$, Matthew P. Cheng ${ }^{1}$, Emily G. McDonald ${ }^{2,3}$ and Todd C. Lee Le, $^{1,23}$
}

\begin{abstract}
Background: Consideration to add empiric MRSA therapy with vancomycin is a common clinical dilemma. However, vancomycin overuse has important adverse events. MRSA colonization screening is commonly performed for infection control. We hypothesized that in cases of S. aureus bacteremia, a score based on patient level factors and MRSA colonization could predict the risk of MRSA infection and inform the need for empiric coverage.

Methods: Using modern machine learning statistical methods (LASSO regression and random forests), we designed a predictive score for MRSA infection based on patient level characteristics, and MRSA colonization as measured by screening done 30 days before infection (30-Day criteria), or at any time before infection (Ever-Positive criteria). Patient factors (age, sex, number of previous admissions, and other medical comorbidities) were obtained through our electronic records.

Results: With random forests, MRSA colonization largely surpassed all other factors in terms of accuracy and discriminatory power. Using LASSO regression, MRSA colonization was the only factor with MRSA infection predictive power with odds ratio of 10.3 (min: 5.99, max: 16.1) and 8.14 (min: 6.01, max: 14.8) for the 30-Day and Ever-Positive criteria, respectively. Further, patient comorbidities were not adequate predictors of MRSA colonization.
\end{abstract}

Conclusions: In an era of community acquired MRSA, colonization status appears to be the only independent and reliable predictor of MRSA infection in cases of $S$. aureus bacteremia. A clinical approach based on a patient's known MRSA colonization status and on local susceptibility patterns may be appropriate.

Keywords: MRSA, Colonization, Vancomycin, Bacteremia, Statistics

\section{Background}

Methicillin resistant Staphylococcus aureus (MRSA) colonization is common in North America [1] and is an important cause of infections including skin and soft tissue, bone and joint, pneumonia and bacteremia. Consideration for empiric MRSA treatment results in patients often being prescribed vancomycin for suspected Gram-positive infections, sometimes days before the final microbiological results are obtained. While this is likely initially appropriate for hemodynamically unstable patients or when the index of suspicion for MRSA is reasonably high, the overuse of vancomycin

\footnotetext{
* Correspondence: guillaume.butler-laporte@mail.mcgill.ca

${ }^{1}$ Division of Infectious Diseases, Department of Medicine, McGill University Health Centre, 1001 Boulevard Décarie, room E05. 1917, Montreal, Quebec H4A 3J1, Canada

Full list of author information is available at the end of the article
}

has a significant potential for harm. It is associated with an increased risk of nephrotoxicity, which may be more common when vancomycin is used in combination with piperacillin-tazobactam [2]. This combination is one of the most commonly used treatments for empiric broad spectrum antimicrobial coverage. Vancomycin-associated nephrotoxicity is associated with an increased length of hospitalization, cost, and an increased risk of mortality [3, 4]. Further, the initial use of vancomycin may delay the timely initiation of beta-lactams, which are superior agents for methicillin sensitive S. aureus (MSSA) [5].

Our group and others have previously demonstrated that MRSA screening swab results can be helpful in determining the probability of MRSA bacteremia in patients presenting with staphylococcal bloodstream

(c) The Author(s). 2018 Open Access This article is distributed under the terms of the Creative Commons Attribution 4.0 International License (http://creativecommons.org/licenses/by/4.0/), which permits unrestricted use, distribution, and 
infection [6]. Other factors associated with MRSA infection such as the presence of chronic wounds, venous catheters, or other in-dwelling medical devices have also been studied in cases of MRSA infection of the bone or joint $[7,8]$.

We hypothesized that by combining individual patient comorbidities and risk factors along with MRSA screening swab results we could better discriminate between MRSA and MSSA bacteremia using statistical algorithms to develop a clinical prediction rule. This would help to avoid potentially inappropriate empiric use of vancomycin.

\section{Methods}

We conducted a retrospective review of all consecutive adult S. aureus bacteremia from April 1, 2010 to April 1, 2015 at the McGill University Health Center (832 beds; 2 hospitals in Montréal, Canada) and obtained their most-recent MRSA screening swab results prior to the blood culture. To prevent patients with multiple positive blood cultures from biasing the results of the study, only the first positive blood culture per patient was included.

Each case of bacteremia was cross-referenced with our electronic medical record to identify associated patient comorbidities in order to calculate the Charlson Comorbidity Index (CCI), a validated scoring system used to assess disease burden and mortality risk [9]. An algorithm was used to calculate the CCI from the ICD-10 codes [10]. We also recorded the number of previous emergency room and inpatient visits within the prior 12 months to serve as a proxy for acute healthcare exposure. All patients on medical, surgical and critical care units are screened for MRSA on admission, and periodically thereafter. A minority of units, such as obstetrics, use only targeted screening. The screen is performed in the nares, though other sites are accepted on a case by case basis. The microbiology protocol has been previously described in detail [6].

MRSA colonization status was interpreted in two ways. To begin, only samples obtained in the 30 days preceding the bacteremia were used to establish recent colonization. Following this, all available screening samples in the patient's history of hospitalizations were used. These were respectively reported as the "30-Day criteria" and the "Ever-Positive criteria" [6].

We used the following two methods for variable selection to predict MRSA bacteremia: [1] random forests methods, and [2] least absolute shrinkage selection and selection operator (Lasso) with 10-fold cross validation and the "one standard error rule" (1SE) [11]. Both methods have their own benefits and pitfalls. Random forests yield good error rates and classifies variables by their accuracy and Gini index (a measure of a variable's ability to discriminate between the potential outcomes), but are harder to interpret. The Lasso provides a reasonable middle ground between interpretability and stability of the effect size estimate [11]. Other variable selection methods exist; however, they tend to be more susceptible to issues involving multiple hypothesis testing and overestimation of effect size (e.g. stepwise regression) or are difficult to interpret clinically (e.g. ridge regression, neural networks). A secondary analysis using Lasso logistic regression was performed to study the variables that most closely predicted MRSA colonization.

Variables were checked for collinearity using variance inflation factors. Analyses were performed in R (v3.2.0) with the glmnet (v2.0-5) and the randomForest (v4.612) packages. The McGill University Health Centre Research Ethics Board approved this study. Consent was not obtained from patients as this was a retrospective study with no intervention. Authorization to release datasets analysed during the current study was not specifically obtained from our ethics board, but can be made available from the corresponding author on reasonable request and in compliance with our REB requirements.

\section{Results}

There were 376 patients with S. aureus bacteremia included, of which 100 (26.6\%) had MRSA. Patient comorbidities are shown in Table 1. Using Random Forest analysis, the five best variables for predicting MRSA bacteremia are presented in Tables 2 and 3 . With both the 30-day and the Ever-Positive criteria, MRSA swabs had a predictive power that was vastly greater than all other variables. For example, a positive MRSA swab had a much greater predictive power than the presence of other seemingly classic risk factors such as age, a history of malignancy, the number of prior hospitalizations in the preceding 12 months, and the Charlson Comorbidity Index. Using the Lasso method and1SE rule (see Additional file 1), the MRSA screening swab was the only variable with any clinically relevant predictive power. Through this method, the logistic regression odds ratios for the 30-Day criteria were on average 10.6 (minimum: 5.08, maximum: 19.2), and for the Ever-Positive criteria on average 8.26 (minimum: 5.23, maximum: 15.8). Using Lasso logistic regression, no patient level comorbidity was predictive of MRSA colonization. This suggests the screening swab is likely the only meaningful variable in the model with respect to clinical prediction. From the $2 \times 2$ tables, we obtained a graphical representation of MRSA swab positive and negative predictive values for MRSA bacteremia (Figs 1 and 2) as functions of prevalence. 
Table 1 Number of patients with each comorbidities (percentage), and median number of admissions to the emergency or a medical ward (interquartile range)

\begin{tabular}{|c|c|c|c|}
\hline Variables & MSSA $(n=276)$ & MRSA $(n=100)$ & $\begin{array}{l}p \text {-value (Fisher's or } \\
\text { Mann-Whitney U) }\end{array}$ \\
\hline Positive MRSA Screen (30 days) & 10/154 (6.49\%) & $52 / 66(78.8 \%)$ & $<0.001$ \\
\hline Positive MRSA Screen (All-Time) & $21 / 215(9.77 \%)$ & 72/91 (79.1\%) & $<0.001$ \\
\hline Median Age (IQR) & $65.5(51,76.25)$ & $66(54.75,77)$ & 0.590 \\
\hline Median CCI (IQR) & $3(1,5)$ & $3(2,4)$ & 0.155 \\
\hline Myocardial Infarction & $39(14.1)$ & $19(19)$ & 0.260 \\
\hline Congestive Heart Failure & $54(19.6)$ & $21(21)$ & 0.771 \\
\hline Peripheral Vascular Disease & $31(11.2)$ & $19(19)$ & 0.059 \\
\hline Cerebrovascular Disease & $18(6.52)$ & $7(7)$ & 0.819 \\
\hline Dementia & $16(5.80)$ & $5(5)$ & 1 \\
\hline Chronic Pulmonary Disease & $53(19.2)$ & $20(20)$ & 0.883 \\
\hline Connective Tissue Disease & $5(1.81)$ & $5(5)$ & 0.139 \\
\hline Peptic Ulcer Disease & $13(4.71)$ & $4(4)$ & 1 \\
\hline Mild Liver Disease & $32(11.6)$ & $9(9)$ & 0.576 \\
\hline Moderate or Severe Liver Disease & $17(6.20)$ & $4(4)$ & 0.612 \\
\hline Diabetes without End-Organ Damage & $80(29.0)$ & $36(36)$ & 0.208 \\
\hline Diabetes with End-Organ Damage & $17(6.16)$ & $10(10)$ & 0.257 \\
\hline Hemiplegia & $12(4.35)$ & $9(9)$ & 0.123 \\
\hline Moderate or Severe Renal Disease & $5(1.81)$ & $15(15)$ & 0.238 \\
\hline Tumor without Metastasis & $34(12.3)$ & $20(20)$ & 0.068 \\
\hline Tumor with Metastasis & $27(9.78)$ & $8(8)$ & 0.691 \\
\hline Leukemia & $16(5.80)$ & $6(6)$ & 1 \\
\hline Lymphoma & $21(7.61)$ & $9(9)$ & 0.669 \\
\hline HIV/AIDS & $3(1.09)$ & $0(0)$ & 0.568 \\
\hline Emergency Visits in Last 6 Months & $1(1,2)$ & $1(1,3)$ & 0.189 \\
\hline Emergency Visits in Last 12 Months & $1(1,2)$ & $2(1,3)$ & 0.258 \\
\hline Inpatient Admissions in Last 6 Months & $0(0,1)$ & $1(0,1)$ & 0.003 \\
\hline Inpatient Admissions in Last 12 Months & $0(0,1)$ & $1(0,2)$ & 0.001 \\
\hline
\end{tabular}

\section{Discussion}

Vancomycin overuse is common in S. aureus bacteremia and may be harmful. Our group and others have previously shown that screening swabs are useful predictors of MRSA bacteremia [6, 12], and could help avoid unnecessary vancomycin prescriptions. However, it is a common belief that MRSA colonization status can be explained by healthcare exposure or the presence of multiple medical comorbidities, and that the swab is a proxy for these comorbidities. Previous studies have discussed several potential predictors of MRSA infection $[7,8]$, but the added benefit of MRSA colonization for

Table 2 Best 5 variables when using random forests with 30-Day criteria, from best to worse

\begin{tabular}{llll}
\hline Variables & Mean Increase in Accuracy (IQR) & Variables & Mean Decrease in Gini (IQR) \\
\hline 30-Day criteria & $73.4(72.2-75.0)$ & 30 -Day Criteria & $35.9(35.7-36.2)$ \\
Tumor without Metastasis & $10.9(10.4-11.5)$ & Age & $9.14(9.05-9.24)$ \\
CCI & $10.2(9.63-10.9)$ & $\mathrm{CCl}$ & $6.69(6.61-6.77)$ \\
Congestive Heart Failure & $6.57(5.94-7.32)$ & Admissions (12 Months) & $5.10(5.03-5.16)$ \\
Dementia & $6.20(5.64-6.78)$ & Myocardial Infarction & $2.06(2.03-2.10)$ \\
\hline
\end{tabular}


Table 3 Best 5 variables when using random forests with Ever-Positive criteria, from best to worse

\begin{tabular}{llll}
\hline Variables & Mean Increase in Accuracy (IQR) & Variables & Mean Decrease in Gini (IQR) \\
\hline Ever-Positive Criteria & $83.2(82.2-84.5)$ & Ever-Positive Criteria & $45.5(45.2-45.9)$ \\
Tumor without Metastasis & $15.6(15.2-16.1)$ & Median Age & $13.3(13.1-13.4)$ \\
Tumor with Metastasis & $9.87(9.42-10.3)$ & Admissions (12 Months) & $8.17(8.10-8.30)$ \\
CCl & $9.76(8.98-10.1)$ & CCl & $7.97(7.89-8.07)$ \\
Diabetes without End-Organ Damage & $9.39(8.75-9.94)$ & Diabetes without End-Organ Damage & $2.83(2.79-2.88)$ \\
\hline
\end{tabular}

clinical decision making was unclear. We examined multiple patient level factors to try and determine which, if any, could predict methicillin resistance, in an effort to develop a clinically meaningful risk score to better optimize the use of anti-MRSA therapy. Our results suggest screening swabs are an independent marker of MRSA infection, regardless of patient comorbidities. The findings of our study are a departure from what has been previously quoted in the literature, in that the only patient level risk factor with a useful clinical predictive ability was a patient's known MRSA status. In fact, the burden of comorbid disease as calculated by the CCI was poorly related to methicillin resistant bacteremia, and both emergency department visits and admissions to acute care failed to contribute significantly.

This outcome may be related to two factors. First, our choice of variable selection technique prioritizes clinical significance over statistical significance. This would explain why variables which were previously reported as statistically related to MRSA did not significantly contribute to predicting infections in our study. Second, over the past 20 years MRSA has become a community acquired organism, and hospital exposure or medical comorbidities may not predict infection risk as well as they once might have. Even patients with minimal healthcare exposure may now be carriers of MRSA.

Three main problems limit our study. First, there were a small number of patients with each individual comorbidity in Table 1 . To alleviate the problem of smaller sample size, we used an established comorbidity score (CCI) and input direct healthcare exposure as separate variables in our model. This continued to support the use of the MRSA screening swab as the sole predictor of MRSA bacteremia. Second, this study is retrospective and therefore hypothesis generating as to whether the use of known MRSA status could optimize vancomycin use with neutral or improved patient outcomes. Third, our institution only regularly screens for MRSA of the nares. While the nares are the most common site for MRSA colonization and correlates well with colonization of other body sites [13], the addition of other body sites would have improved our test sensitivity, though the

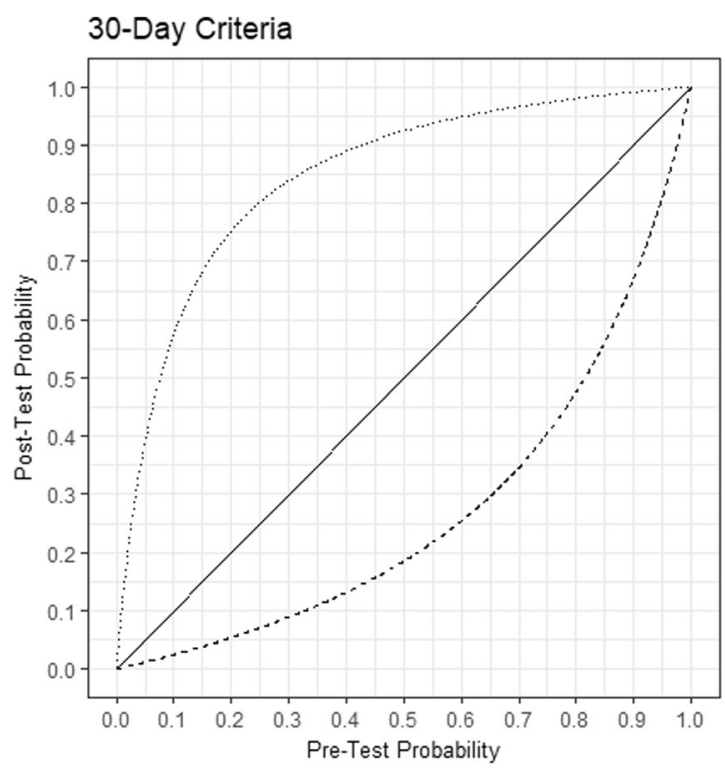

- - Negative Test Result

.... Positive Test Result

Fig. 1 Post-test probability of the 30-Day criteria, as a function of pre-test probability 


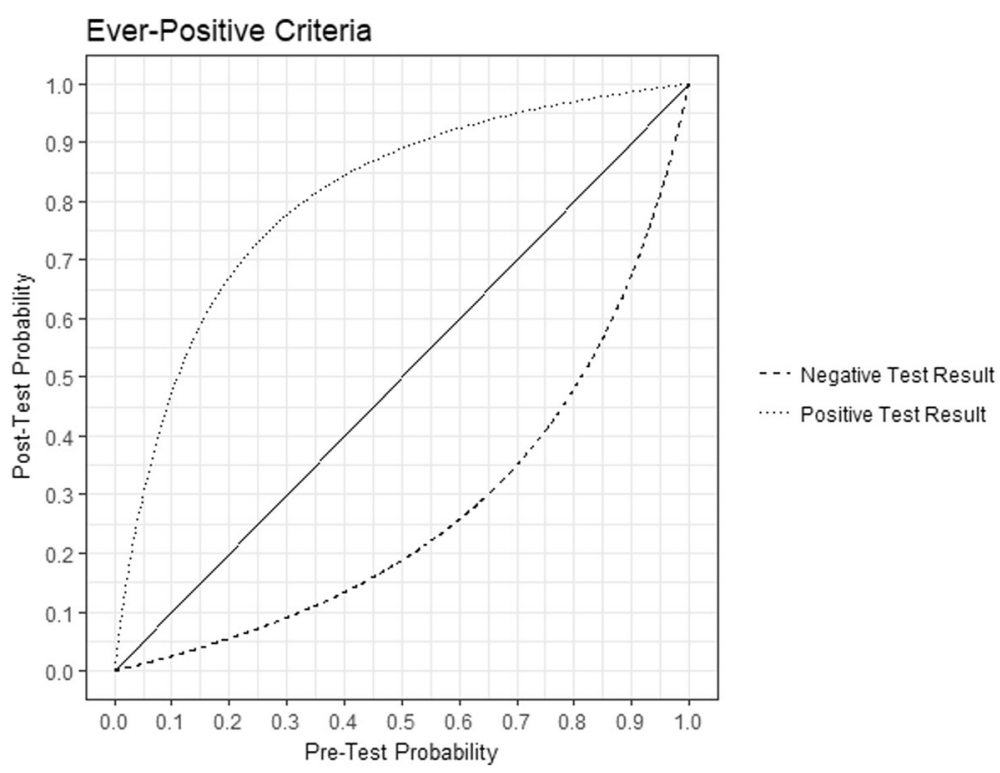

Fig. 2 Post-test probability of the Ever-Positive criteria, as a function of pre-test probability

incurring loss of specificity is hard to establish. However, it is likely the overall effect would be an improvement of the negative predictive value with a reduction in the positive predictive value. Given the simplicity of the clinical question, and the fact that the data required for the analysis can be collected retrospectively without introducing significant bias, we still believe that our results could be helpful for clinicians who want to estimate the risk of MRSA bloodstream infection.

First, depending on the local prevalence of methicillin resistance in $S$. aureus bacteremia and in the absence of a nosocomial MRSA outbreak, MRSA colonization status could be factored into the clinical decision to initiate intravenous vancomycin in stable patients and antistaphylococcal beta-lactams in all patients so as to limit both under and overtreatment [6]. Second, we should reconsider whether MRSA colonization status is directly linked to the usual medical comorbidities. Based on our results, variables that were previously thought to be associated with MRSA such as healthcare exposure may not be dependable factors to infer the presence of drug resistant bacteria. The notion that healthcare exposure is a consistently reliable marker for the presence of resistant organisms is being challenged, as was illustrated in recent IDSA guidelines with the removal of the classification of "Healthcare-Associated" pneumonia [14]. In an era of community acquired resistant organisms such as MRSA, our results suggest that favoring an approach based on known colonization status and local susceptibility patterns may be more appropriate.

\section{Conclusions}

We have demonstrated that known MRSA colonization status, along with knowledge of local prevalence patterns, is a more powerful prediction tool than any other patient level comorbidity in guiding early and appropriate treatment in Staphylococcus aureus bacteremia. We hope that prospective studies will demonstrate that in appropriately selected hemodynamically stable patients this approach reduces the overuse of potentially toxic therapies, increases early targeted beta-lactam therapy, and improves patient outcomes.

\section{Additional file}

Additional file 1: Lasso Analysis An example of the data analysis performed using the Lasso method. The following are representative curve obtained from repeating the analysis 100 times. Figure S1A. Patient level comorbidities and 30-Day criteria vs MRSA bacteremia. Figure S1B. Patient level comorbidities and Ever-Positive criteria vs MRSA bacteremia (DOCX 2607 kb).

\section{Abbreviations}

1SE: 1 standard error rule; CCl: Charlson comorbidity index; MRSA: Methicillin resistant Staphylococcus aureus; MSSA: Methicillin sensitive Staphylococcus aureus

\section{Availability of data and materials}

Authorization to release datasets analysed during the current study was not specifically obtained from our ethics board. Data would be available from the corresponding author on reasonable request with REB approval and a data sharing agreement.

\section{Author's contributions}

GBL analyzed the dataset it. GBL, MPC, EGM, TCL were involved in designing the study and interpretation of the data and its analysis. All authors were involved in writing and editing the manuscript. All authors read and approved the final manuscript. 


\section{Ethics approval}

Obtained from McGill University Health Center Research Ethics Board, which also deemed it unnecessary to obtain consent directly from patients as this was a retrospective study with no intervention.

\section{Competing interests}

The authors declare that they have no competing interests.

\section{Publisher's Note}

Springer Nature remains neutral with regard to jurisdictional claims in published maps and institutional affiliations.

\section{Author details}

'Division of Infectious Diseases, Department of Medicine, McGill University Health Centre, 1001 Boulevard Décarie, room E05. 1917, Montreal, Quebec H4A 3J1, Canada. ${ }^{2}$ Division of General Internal Medicine, Department of Medicine, McGill University Health Centre, Montreal, Quebec, Canada.

${ }^{3}$ Clinical Practice Assessment Unit, McGill University Health Centre, Montreal, Quebec, Canada.

Received: 3 January 2018 Accepted: 3 June 2018

Published online: 11 June 2018

\section{References}

1. Jarvis WR, Jarvis AA, Chinn RY. National prevalence of methicillin-resistant Staphylococcus aureus in inpatients at United States health care facilities, 2010. Am J Infect Control. 2012;40:194-200.

2. Navalkele B, Pogue JM, Karino S, Nishan B, Salim M, Solanki S, Pervaiz A Tashtoush N, Shaikh H, Koppula S, Koons J, Hussain T, Perry W, Evans R, Martin ET, Mynatt RP, Murray KP, Rybak MJ, Kaye KS. Risk of acute kidney injury in patients on concomitant vancomycin and piperacillinTazobactam compared to those on vancomycin and Cefepime. Clin Infect Dis. 2017;64:116-23.

3. Pritchard L, Baker C, Leggett J, Sehdev P, Brown A, Bayley KB. Increasing vancomycin serum trough concentrations and incidence of nephrotoxicity. Am J Med. 2010;123:1143-9.

4. Jeffres MN. The whole price of vancomycin: toxicities, troughs, and time. Drugs. 2017;77:1143-54.

5. Chang FY, Peacock JE Jr, Musher DM, Triplett P, MacDonald BB, Mylotte JM, O'Donnell A, Wagener MM, Yu VL. Staphylococcus aureus bacteremia: recurrence and the impact of antibiotic treatment in a prospective multicenter study. Medicine (Baltimore). 2003;82:333-9.

6. Butler-Laporte G, Cheng MP, Cheng AP, MCDonald EG, Lee TC. Using MRSA screening tests to predict methicillin resistance in Staphylococcus aureus bacteremia. Antimicrob Agents Chemother. 2016;60:7444-8.

7. Epstein L, Mu Y, Belflower R, Scott J, Ray S, Dumyati G, Felsen C, Petit S, Yousey-Hindes K, Nadle J, Pasutti L, Lynfield R, Warnke L, Schaffner W, Leib K, Kallen AJ, Fridkin SK, Lessa FC. Risk factors for invasive methicillin-resistant Staphylococcus aureus infection after recent discharge from an acute-care hospitalization, 2011-2013. Clin Infect Dis. 2016;62:45-52.

8. Lee JY, Chong YP, Kim T, Hong HL, Park SJ, Lee ES, Kim MN, Kim SH, Lee SO, Choi $\mathrm{SH}$, Woo JH, Kim YS. Bone and joint infection as a predictor of community-acquired methicillin-resistant Staphylococcus aureus bacteraemia: a comparative cohort study. J Antimicrob Chemother. 2014;69: 1966-71.

9. Charlson ME, Pompei P, Ales KL, MacKenzie CR. A new method of classifying prognostic comorbidity in longitudinal studies: development and validation. J Chronic Dis. 1987;40:373-83.

10. Quan H, Sundararajan V, Halfon P, Fong A, Burnand B, Luthi JC, Saunders $L D$, Beck CA, Feasby TE, Ghali WA. Coding algorithms for defining comorbidities in ICD-9-CM and ICD-10 administrative data. Med Care. 2005; 43:1130-9.

11. Hastie T, Tibshirani R, Friedman J. The elements of statistical learning: data mining, inference, and prediction. New York: Second ed: Springer; 2009. https://www.springer.com/la/book/9780387848570

12. MacFadden DR, Elligsen M, Robicsek A, Ricciuto DR, Daneman N. Utility of prior screening for methicillin-resistant Staphylococcus aureus in predicting resistance of S. Aureus infections. Cmaj. 2013;185:E725-30.

13. Mermel LA, Cartony JM, Covington P, Maxey P, Morse D. Methicillin-resistant Staphylococcus aureus colonization at different body sites: a prospective, quantitative analysis. J Clin Microbiol. 2011;49:1119-21.
14. Kalil AC, Metersky ML, Klompas M, Muscedere J, Sweeney DA, Palmer LB, Napolitano LM, O'Grady NP, Bartlett JG, Carratalà J, El Solh AA, Ewig S, Fey PD, File TM, Restrepo MI, Roberts JA, Waterer GW, Cruse P, Knight SL, Brozek JL. Management of Adults with Hospital-acquired and Ventilator-associated Pneumonia: 2016 clinical practice guidelines by the Infectious Diseases Society of America and the American Thoracic Society. Clin Infect Dis. 2016; 63:e61-111. https://doi.org/10.1093/cid/ciw353.

\section{Ready to submit your research? Choose BMC and benefit from:}

- fast, convenient online submission

- thorough peer review by experienced researchers in your field

- rapid publication on acceptance

- support for research data, including large and complex data types

- gold Open Access which fosters wider collaboration and increased citations

- maximum visibility for your research: over $100 \mathrm{M}$ website views per year

At BMC, research is always in progress.

Learn more biomedcentral.com/submissions 